\title{
Simplicial edge representation of protein structures and alpha contact potential with confidence measure
}

\author{
Xiang Li, Changyu Hu and Jie Liang* \\ Department of Bioengineering, SEO, MC-063 \\ University of Illinois at Chicago \\ 851 S. Morgan Street, Room 218 \\ Chicago, IL 60607-7052, U.S.A. \\ (Accepted by Proteins.)
}

October 22, 2018

\begin{abstract}
Protein representation and potential function are two important ingredients for studying proteins folding, equilibrium thermodynamics, and sequence design. We introduce a novel geometric representation of protein contact interactions using the edge simplices from alpha shape of protein structure. This representation can eliminate implausible neighbors that are not in physical contact, and can avoid spurious contact between two residues when a third residue is between them. We develop statistical alpha contact potential using an odds-ratio model. A studentized bootstrap method is then introduced for assessing the 95\% confidence intervals for each of the 210 propensity parameters. We found with confidence that there is significant long range propensity ( $>30$ residues apart) for hydrophobic interactions. We test alpha contact potential for native structure discrimination using several sets of decoy structures, and found it often has comparable performance with atom-based potentials requiring many more parameters. We also show that accurate geometric representation is important, and alpha contact potential has better performance than potential defined by cut-off distance between geometric centers of side chains. Hierarchical clustering of alpha contact potentials reveals natural grouping of residues. To explore the relationship between shape representation and physicochemical representation, we test the minimum alphabet size necessary for native structure discrimination. We found that there is no significant difference in performance
\end{abstract}

\footnotetext{
*Corresponding author. Phone: (312)355-1789, fax: (312)996-5921, email: jliang@uic.edu
}

of discrimination when alphabet size varies from 7 to 20, if geometry is represented accurately by alpha simplicial edges. This result suggests that the geometry of packing plays an important role, but the specific residue types are often interchangeable.

Keywords: Simplicial edge; alpha contact; alpha shape; protein potential function; bootstrap;

\section{Introduction}

Potential function plays important roles for a variety of computational studies of proteins, including prediction of protein structures, characterization of ensemble thermodynamic properties of proteins, and design of novel proteins. For example, an essential requirement for the prediction of three-dimensional structure of protein from primary sequence is a potential function which can select the native conformation from an ensemble of alternative conformations. Potential function is also often used to guide the sampling of protein conformations [1]. A variety of potential functions have been developed for these important tasks, including physical model based potentials [2-4], empirical statistical potentials [5-7], and empirical potentials obtained from optimization [8-12].

The effectiveness of potential function depends on another critically important factor, i.e., the representation of protein structures. Within this framework, we explore a new type of pairwise contact potentials using a novel contact definition. We introduce a contact definition that reflects protein geometry more accurately. These contacts are based on the computa- 
tion of the alpha shape, or the dual simplicial complex description of protein structures $[13,14]$. Here contact occurs if atoms from non-bonded residues share a Voronoi edge, and this edge is at least partially contained in the body of the molecule. In another word, atoms have to be in physical nearest neighbor contact. In this study, we only examine the 1-simplices, or edges in the dual simplicial complex, which represents the pairwise contacts. This description is related to the work from Wodak's group, where the contact area between atoms are calculated as the area of intersection of the accessible atom ball around each atom and the faces of its weighted Voronoi cell [15].

We develop a statistical contact propensities based on alpha edge simplices and a combinatorial null model. To account for the uncertainty of estimated propensity parameter, we develop a studentized bootstrap method for estimating their $95 \%$ confidence intervals. We also examine how geometric representation of dual simplicial complex influence the effectiveness of pairwise contact potential functions. In addition, we aim to understand how the size of alphabet of amino acid residues affect the effectiveness of empirical pair potentials. This is important for protein design, where any reduction of the alphabet size of residues will result in exponentially more efficient sampling in the sequence space, therefore leading to more successful design strategies $[16,17]$.

This work is also motivated by the need to develop potential functions that takes advantage of recent development in computational geometry and computational topology. The alpha shape representation of proteins from computational geometry allow rapid calculations of metric, topological, and combinatorial structures of proteins precisely $[14,18,19]$. These advantages can lead to improvements in voids and binding site detection [18-20], in hierarchical representation of protein dynamic shapes at different resolution, and in conformation sampling. Recent theoretical development suggests many intriguing applications in protein studies [21-23]. These important advances are largely unexploited currently, and we hope this work provides a useful link by developing empirical pairwise contact potentials based on dual simplicial complex representations of proteins.

Our approach also solves a problem that cannot be satisfactorily addressed with current contact pair potentials. In current approaches, pairwise contact interactions are declared if two residues are within a specific cut-off distance. Potentials based on this contact definition have achieved considerable success.
Nevertheless, contacts by distance cut-off can potentially include many implausible non-contacting neighbors, which have no significant physical interaction [24]. Whether or not a pair of residues can make physical contact depends not only on the distance between their center positions (such as $\mathrm{C}_{\alpha}$ or $\mathrm{C}_{\beta}$, or geometric centers of side chain), but also on the size and the orientations of side-chains [24]. Furthermore, two atoms close to each other may in fact be shielded from contact by other atoms. As emphasized in [25], these contact pairs should not contribute to the assessment of pairwise contacts. By occupying the intervening space, other residues can block a pair of residues from interacting with each other. Inclusion of these fictitious contact interactions would be undesirable.

We organized this paper as follows. First, we describe briefly the dual simplicial complex representation of proteins structures. We then discuss the probabilistic models for developing pairwise potentials. A bootstrap resampling procedure is then introduced which provide confidence intervals of estimated pairwise potential parameters. We then present the pairwise contact potential along with experimental results in discriminating native structure against decoy structures using several benchmark data sets. We further examine how pair potentials developed from the dual simplices compare with cut-off contact definitions using side-chain centers. The effects of reduced alphabet size for amino acid residues are then described. We conclude with remarks and discussion.

\section{Model and Methods}

Alpha Contacts from Dual Simplicial Complex. Alpha shape has been successfully applied to study a number of problems in proteins, including void measurement, binding site characterization, protein packing, electrostatic calculations, and protein hydrations [14,18, 20,26-30]. Details of alpha shape have been described elsewhere, here we only provide a brief description for completeness.

To illustrate, Figure 1a shows a two-dimensional molecule formed by a collection of disks of uniform size. Each Voronoi cell is defined by its boundaries, shown as broken lines. Every Voronoi edge is a perpendicular bisector of the line between two atom centers. Each Voronoi cell contains one atom, and every point inside a Voronoi cell is closer to this atom than to any other atom. Three connected Voronoi 


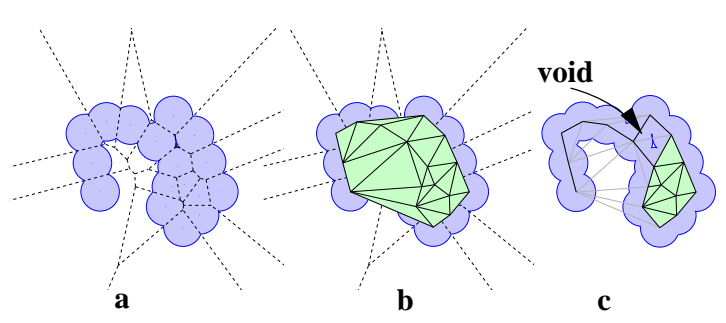

Figure 1: Geometric constructs of a simple 2D molecule. a) The molecule is formed by disks of uniform size. The dashed lines represent the Voronoi diagram, where each region contains one atom. b) The convex hull of the atom centers, and the Delaunay triangulation of the convex hull. c) The alpha shape of the 2D molecule is a subset of the Delaunay triangulation. It is contained within the molecule, and reflects the topological and metric properties of the molecule.

edges meet at a Voronoi vertex. Another geometric construct, the Delaunay triangulation (Fig 1b) is mathematically dual to the Voronoi diagram, and can be explained by the following procedure. For each Voronoi edge, connect the corresponding two atom centers with a line segment, and for each Voronoi vertex, place a triangle spanning the three atom centers of the three Voronoi cells. Completing this for all Voronoi edges and Voronoi vertices gives a collection of line segments and triangles. Together with the vertices representing atom centers, they form the "Delaunay complex", which is the underlying structure of Delaunay triangulation.

Now we remove all Delaunay edges (or line segments) where corresponding Voronoi edge of the two atoms does not intersect with the molecular (Figure 1c)). When two atoms are spatially very close, the balls representing the two atoms intersect, and these two atoms have non-zero, two-body volume overlap. When three atoms are spatially very close, they intersect and have non-zero, three-body volume overlap. We further remove all Delaunay triangles where the corresponding Voronoi vertex of the three atoms is not contained within the molecule. The subset of the Delaunay complex formed by the remaining triangles, edges and vertices (atom centers) is called the dual simplicial complex, or the alpha complex. We are interested in identifying only contacting atoms that are spatial nearest neighbors. These are precisely atoms with two-body volume overlaps whose Voronoi cells intersect. By following the mathematical dual structure, i.e., the edges in the alpha complex, we can accurately identify all contacting nearest neighboring atom pairs. For convenience, we use a rather arbitrary criterion and declare two residues are in alpha contact if there is at least one edge connecting these two residues.

Using the alpha shape API kindly provided by Prof. Edelsbrunner, a program INTERFACE has been implemented to compute contacting atoms based on precomputed Delaunay triangulation and alpha shape. The Delaunay triangulation is computed using the DELCX program, and the alpha shapes computed using the MKALF program. Both can be downloaded from the website at NCSA (http://www.ncsa.uiuc.edu). The van der Waals radii of protein atoms are taken from [31]. We follow Singh \& Thornton and increment the van der Waals radii by $0.5 \AA[32]$. This increment is small and comparable with the resolution of the structure. It enables the modeling of imprecisely determined atomic coordinates without introducing many spurious twobody contacts.

Probabilistic Model for Pairwise Alpha Contact Propensity. The propensity $P(i, j)$ for residue of type $i$ interacting with residue of type $j$ is modeled as the odds ratio of the observed probability $q(i, j)$ and the expected probability $p(i, j)$ of a pairwise alpha contact involving both residue $i$ and $j,:$

$$
P(i, j)=\frac{q(i, j)}{p(i, j)}
$$

To compute $p(i, j)$ and $q(i, j)$, we choose a simple null model, where the observed contacts of different proteins in the entire database are pooled together, and the expected contact numbers are calculated. This is the same as the reference state of compositionindependent scale discussed in literature [33]. We have:

$$
q(i, j)=\frac{a(i, j)}{\sum_{i^{\prime}, j^{\prime}} a\left(i^{\prime}, j^{\prime}\right)}
$$

Here, $a(i, j)$ is the number count of atomic contacts between residue type $i$ and residue type $j$, and $\sum_{i^{\prime}, j^{\prime}} a\left(i^{\prime}, j^{\prime}\right)$ is the total number of all atomic contacts. The observed probability is then compared against the random probability $p(i, j)$ that a pair of contacting atoms is picked from a residue of type $i$ and a residue of type $j$, when chosen randomly and independently [34]. We have:

$p(i, j)=N_{i} N_{j} \cdot\left(\frac{n_{i} n_{j}}{n\left(n-n_{i}\right)}+\frac{n_{i} n_{j}}{n\left(n-n_{j}\right)}\right), \quad$ when $\quad i \neq j$ and

$$
p(i, j)=N_{i} N_{i-1} \cdot \frac{n_{i} n_{i}}{n\left(n-n_{i}\right)}, \quad \text { when } \quad i=j
$$


where $N_{i}$ is the number of interacting residues of type $i, n_{i}$ is the number of atoms residue of type $i$ has, and $n$ is the total number of interacting atoms.

The alpha contact potential between residue $i$ and residue $j$ is obtained from the propensity value $P(i, j)$ as $\ln P(i, j)$, and the overall energy of a protein is calculated as:

$$
\epsilon=-\sum_{i, j} \ln P(i, j)
$$

Cys-Cys Interactions. In principle, only 210 parameters are necessary for 20 amino acid residues. However, Cys-Cys contact requires special attention. Its propensity value is the largest compared to the other 209 contacts, because Cys residue tends to form disulfide bond with another Cys residue. Nevertheless, there are many Cys-Cys residue pairs that are in close spatial proximity but do not form disulfide bond. As a result, a mis-classification of a nondisulfide Cys-Cys contact as a disulfide bond will affect the overall score considerably, especially for small proteins with abundant Cys residues. The problem associated with the mis-classification of Cys-Cys contact was already pointed out in [10].

To avoid assigning the same score to the two different types of Cys-Cys contacts, we introduce a slightly more detailed propensity scores for Cys-Cys interactions. Since contacts between C:O, C:N, C:C, and $\mathrm{O}: \mathrm{O}$ atoms never appear in disulfide bonds, a CysCys contact pair lacking these atomic interactions is classified as a disulfide bond Cys-Cys contact if in addition the distance between their SG atoms is less than $2.5 \AA$. All other Cys-Cys interactions are classified as nonbonded Cys-Cys contact. The propensity values estimated for these two types of Cys-Cys contacts are listed in Table II.

Bootstrap Resampling. Because the sample size of 1,045 proteins in PDBSELECT is limited, statistical modeling with approximations may be prone to errors. It is therefore essential to assess reliability of estimated contact potentials. Here we apply bootstrap techniques to calculate confidence intervals from simulated data sets $[35,36]$. For alpha contact potential of a specific residue pair, (e.g., Trp-Trp), we denote the true value of the contact potential as $\theta$. Our probabilistic model (Equation 1) can be regarded as an estimator $T$ that gives the estimated value $t$ from the finite amount of data for $\theta$. Our goal is to calculate a $95 \%$ confidence interval for $\theta$.
We resample 1,045 proteins independently $R$ times from the set of 1,045 proteins from PDBSELECT, with replacement allowed. We have a simulated data set of $Y_{1}^{*}, . ., Y_{R}^{*}$, each contains 1,045 proteins. Some structures in the original PDBSELECT set appear multiple times, some appear once, and some never appear. We estimate the pair contact value $\theta$ from each of the $R$ samples, and obtain $t_{1}^{*}, . ., t_{R}^{*}$. For an equitailed $95 \%$ confidence interval $(95 \%=1-2 \alpha, \alpha=2.5 \%)$, we have the basic bootstrap confidence limits:

$$
\left(t_{(R+1)(1-\alpha)}^{*}, \quad t_{(R+1) \alpha}^{*}\right)
$$

The accuracy of these limits depend on $R$, and how well the distribution of $\left\{t^{*}-t\right\}$ agrees with that of $T-\theta$. Perfect agreement occurs only when the distribution of $T-\theta$ does not depend on any unknown variables.

To reduce possible errors due to unknown variables, we use studentized bootstrap. For the $r$ th bootstrapped sample, we calculate:

$$
z_{r}^{*}=\frac{t_{r}^{*}-t}{v_{r}^{* 1 / 2}}
$$

To obtain $v_{r}^{*}$, we bootstrap with replacement again $M$ times the $r$ th sample of the original bootstrap. We have:

$$
v_{r}^{*}=\frac{1}{M-1} \sum_{m=1}^{M}\left(t_{m}^{*}-\bar{t}^{*}\right)^{2}
$$

where $t_{1}^{*}, \ldots t_{M}^{*}$ are calculated from the second bootstrap sampling. We then use the $(R+1) \cdot \alpha$ th order statistic of the simulated values $z_{1}^{*}, \ldots, z_{R}^{*}$, or $z_{(R+1) \alpha}^{*}$ to obtain the studentized bootstrap confidence interval for $\theta$ :

$$
\left(t-v^{1 / 2} z_{(R+1)(1-\alpha)}^{*}, \quad t-v^{1 / 2} z_{(R+1) \alpha}^{*}\right)
$$

Since $M$ bootstrap samples from the $r$ th sample are needed to obtain $v_{r}^{*}$, the total number of nested bootstrap samples is $(M+1) \cdot R$. We chose $R=1,000$ and $M=50$. Altogether, we generate $1,001 \times 50=$ 50,050 bootstrap samples to calculate the confidence intervals for each of the $209+2$ pairwise alpha contact propensities.

Database Selection. In this study, we use PDbSELECT from http://www.cmbi.kun.nl /swift/pdbsel [37,38]. PDBSELECT contains 1,045 proteins selected from the Protein Data Bank (PDB). The sequence identity between any pair of proteins in PDBSELECT is smaller than $25 \%$. 


\begin{tabular}{|c|c|c|c|c|c|c|c|c|c|c|}
\hline & ALA & $\mathrm{ARG}$ & ASN & $\mathrm{ASP}$ & CYS & GLN & GLU & GLY & HIS & ILE \\
\hline ALA & 2.1 & 1.0 & 0.9 & 0.8 & 1.7 & 1.0 & 0.8 & 1.4 & 1.0 & 1.7 \\
\hline ARG & $0 . \overline{9-1.0}$ & $\underline{0.7}$ & 0.8 & 1.2 & 0.8 & 0.9 & 1.3 & 0.9 & 0.8 & 0.8 \\
\hline ASN & $0.9-1.1$ & $0 . \overline{7-0} .8$ & $\underline{1.0}$ & 0.9 & 0.8 & 0.9 & 0.7 & 1.0 & 0.8 & 0.7 \\
\hline ASP & $0.8-0.9$ & $1.1-1.3$ & $0 . \overline{9-1.0}$ & $\underline{0.6}$ & 0.7 & 0.8 & 0.5 & 0.8 & 0.8 & 0.6 \\
\hline CYS & $1.5-1.9$ & $0.7-0.9$ & $0.7-0.9$ & $0.6-0.9$ & ${ }^{a} \underline{\underline{15.2}}$ & 0.8 & 0.6 & 1.5 & 1.3 & 1.5 \\
\hline GLN & $1.0-1.1$ & $0.8-0.9$ & $0.8-1.0$ & $0.7-0.8$ & $0 . \overline{7-0.9}$ & $\underline{0.9}$ & 0.8 & 0.9 & 0.7 & 0.8 \\
\hline GLU & $0.8-0.9$ & $1.2-1.4$ & $0.6-0.7$ & $0.4-0.5$ & $0.5-0.7$ & $0.7-0.8$ & $\underline{0.5}$ & 0.6 & 0.7 & 0.7 \\
\hline GLY & $1.3-1.5$ & $0.9-1.0$ & $1.0-1.1$ & $0.8-0.9$ & $1.3-1.7$ & $0.8-1.0$ & $0.6-0.7$ & $\underline{1.5}$ & 0.8 & 1.1 \\
\hline HIS & $0.9-1.1$ & $0.7-0.9$ & $0.7-0.8$ & $0.8-0.9$ & $1.1-1.5$ & $0.6-0.8$ & $0.6-0.7$ & $0.7-0.9$ & $\underline{1.0}$ & 0.9 \\
\hline ILE & $1.5-1.9$ & $0.7-0.8$ & $0.7-0.8$ & $0.6-0.6$ & $1.4-1.7$ & $0.7-0.9$ & $0.6-0.7$ & $1.0-1.2$ & $0.8-0.9$ & $\underline{2.1}$ \\
\hline LEU & $1.5-1.6$ & $0.8-0.9$ & $0.7-0.8$ & $0.6-0.7$ & $1.4-1.7$ & $0.9-1.0$ & $0.7-0.7$ & $1.0-1.2$ & $1.0-1.1$ & $1 . \overline{9-2.0}$ \\
\hline LYS & $0.8-0.9$ & $0.4-0.5$ & $0.7-0.9$ & $1.2-1.3$ & $0.5-0.7$ & $0.7-0.8$ & $1.3-1.4$ & $0.7-0.8$ & $0.5-0.7$ & $0.7-0.8$ \\
\hline MET & $1.5-1.7$ & $0.8-1.0$ & $0.7-0.9$ & $0.6-0.8$ & $1.5-2.2$ & $0.9-1.1$ & $0.7-0.8$ & $1.1-1.4$ & $0.9-1.2$ & $1.7-2.1$ \\
\hline PHE & $1.2-1.4$ & $0.8-1.0$ & $0.7-0.9$ & $0.5-0.6$ & $1.5-1.9$ & $0.8-0.9$ & $0.6-0.6$ & $1.0-1.2$ & $0.9-1.1$ & $1.5-1.7$ \\
\hline PRO & $0.8-0.9$ & $0.7-0.8$ & $0.5-0.7$ & $0.4-0.5$ & $1.0-1.3$ & $0.7-0.8$ & $0.5-0.6$ & $0.8-0.9$ & $0.7-0.9$ & $0.7-0.8$ \\
\hline SER & $1.1-1.2$ & $0.7-0.8$ & $0.9-1.0$ & $1.0-1.1$ & $1.1-1.5$ & $0.8-1.0$ & $0.8-0.9$ & $1.1-1.2$ & $0.9-1.1$ & $0.8-0.9$ \\
\hline THR & $1.1-1.3$ & $0.8-0.9$ & $0.9-1.0$ & $0.8-1.0$ & $0.9-1.2$ & $0.9-1.1$ & $0.8-0.9$ & $1.0-1.2$ & $0.8-1.0$ & $1.0-1.1$ \\
\hline TRP & $1.0-1.2$ & $1.2-1.5$ & $0.8-1.1$ & $0.5-0.7$ & $1.4-2.1$ & $0.9-1.2$ & $0.6-0.8$ & $1.1-1.4$ & $1.2-1.6$ & $1.2-1.5$ \\
\hline TYR & $1.0-1.2$ & $1.0-1.1$ & $0.7-0.8$ & $0.7-0.7$ & $1.2-1.5$ & $0.8-1.0$ & $0.6-0.7$ & $1.0-1.2$ & $1.0-1.3$ & $1.2-1.4$ \\
\hline VAL & $1.5-1.7$ & $0.7-0.8$ & $0.7-0.8$ & $0.5-0.6$ & $1.4-1.7$ & $0.7-0.8$ & $0.6-0.7$ & $1.1-1.2$ & $0.8-0.9$ & $1.8-1.9$ \\
\hline${ }^{b} C I_{(i, i)}$ & $1.9-2.3$ & $0.7-0.8$ & $0.9-1.1$ & $0.5-0.7$ & $13.2-17.3$ & $0.8-1.0$ & $0.5-0.5$ & $1.4-1.6$ & $0.8-1.2$ & $2.0-2.2$ \\
\hline & LEU & $\overline{L Y S}$ & MET & PHE & $\mathrm{PRO}$ & SER & THR & TRP & TYR & $\overline{\text { VAL }}$ \\
\hline ALA & 1.6 & 0.9 & 1.6 & 1.2 & 0.8 & 1.2 & 1.2 & 1.1 & 1.1 & 1.6 \\
\hline ARG & 0.9 & 0.5 & 0.9 & 0.9 & 0.7 & 0.8 & 0.8 & 1.3 & 1.1 & 0.7 \\
\hline ASN & 0.7 & 0.8 & 0.8 & 0.8 & 0.6 & 1.0 & 0.9 & 1.0 & 0.8 & 0.7 \\
\hline ASP & 0.6 & 1.3 & 0.7 & 0.5 & 0.5 & 1.0 & 0.9 & 0.6 & 0.7 & 0.6 \\
\hline CYS & 1.6 & 0.6 & 1.8 & 1.7 & 1.1 & 1.3 & 1.1 & 1.8 & 1.4 & 1.5 \\
\hline GLN & 1.0 & 0.8 & 1.0 & 0.8 & 0.8 & 0.9 & 1.0 & 1.0 & 0.9 & 0.8 \\
\hline GLU & 0.7 & 1.3 & 0.7 & 0.6 & 0.6 & 0.8 & 0.8 & 0.7 & 0.6 & 0.6 \\
\hline GLY & 1.1 & 0.8 & 1.2 & 1.1 & 0.9 & 1.2 & 1.1 & 1.2 & 1.1 & 1.2 \\
\hline HIS & 1.0 & 0.6 & 1.1 & 1.0 & 0.8 & 1.0 & 0.9 & 1.4 & 1.1 & 0.9 \\
\hline ILE & 1.9 & 0.7 & 1.9 & 1.6 & 0.7 & 0.9 & 1.0 & 1.3 & 1.3 & 1.8 \\
\hline LEU & $\underline{2.0}$ & 0.7 & 1.8 & 1.7 & 0.7 & 0.9 & 1.0 & 1.6 & 1.3 & 1.7 \\
\hline LYS & $0.7-0.7$ & $\underline{0.5}$ & 0.7 & 0.7 & 0.5 & 0.8 & 0.7 & 0.9 & 0.9 & 0.7 \\
\hline MET & $1.7-1.9$ & $0.7-0.8$ & $\underline{2.4}$ & 1.9 & 0.9 & 1.0 & 1.1 & 1.6 & 1.5 & 1.6 \\
\hline PHE & $1.7-1.8$ & $0.7-0.8$ & $1 . \overline{7-2.0}$ & $\underline{2.0}$ & 1.0 & 1.0 & 0.8 & 1.7 & 1.4 & 1.6 \\
\hline PRO & $0.7-0.8$ & $0.5-0.6$ & $0.8-1.0$ & $0.9-1.0$ & $\underline{0.7}$ & 0.7 & 0.7 & 1.4 & 1.2 & 0.8 \\
\hline SER & $0.9-1.0$ & $0.7-0.8$ & $0.9-1.2$ & $0.9-1.0$ & $0 . \overline{6}-0.8$ & $\underline{1.1}$ & 1.0 & 1.0 & 0.9 & 0.9 \\
\hline THR & $0.9-1.0$ & $0.6-0.7$ & $1.0-1.2$ & $0.8-0.9$ & $0.6-0.7$ & $1.0-1.1$ & $\underline{1.1}$ & 0.8 & 0.8 & 1.0 \\
\hline TRP & $1.5-1.7$ & $0.8-1.0$ & $1.4-1.9$ & $1.5-1.8$ & $1.3-1.5$ & $0.9-1.1$ & $0.8-0.9$ & $\underline{1.8}$ & 1.4 & 1.4 \\
\hline TYR & $1.3-1.4$ & $0.9-1.0$ & $1.4-1.6$ & $1.3-1.5$ & $1.1-1.3$ & $0.8-0.9$ & $0.8-0.9$ & $1.3-1.5$ & $\underline{1.2}$ & 1.2 \\
\hline VAL & $1.7-1.8$ & $0.6-0.7$ & $1.5-1.7$ & $1.5-1.6$ & $0.8-0.9$ & $0.8-0.9$ & $1.0-1.1$ & $1.3-1.5$ & $1.1-1.3$ & $\underline{1.8}$ \\
\hline$C I_{(i, i)}$ & $1.9-2.1$ & $0.5-0.5$ & $2.1-2.7$ & $1.8-2.1$ & $0.6-0.8$ & $1.1-1.3$ & $1.0-1.2$ & $1.5-2.1$ & $1.1-1.3$ & $1.7-1.9$ \\
\hline${ }^{c} P(\mathrm{CC})$ & \multicolumn{10}{|c|}{$P_{\text {non-disulfide-bond }}=1.8$ with $C I=(1.7,1.9) ;$} \\
\hline
\end{tabular}

Table I: The alpha contact potential of pairwise interactions of amino acid residues. The upper triangle of the table lists the propensity values, the lower triangle lists the $95 \%$ confidence intervals. The $95 \%$ confidence intervals for the diagonal entries are listed separately. The potential values for the two different types of Cys-Cys contacts are also listed.

${ }^{a}$ The alpha contact potential of Cys-Cys if all Cys-Cys conformations are classified as one type

${ }^{b} 95 \%$ confidence interval of alpha contact potentials between self-pair of amino acid residues.

${ }^{c} P_{\text {non-disulfide-bond }}$ is the potential of Cys-Cys without a disulfide bond; $P_{\text {disulfide-bond }}$ is the potential of Cys-Cys with a disulfide bond.

\section{Results}

Pairwise Alpha Contact Potentials. The pairwise alpha contact propensities are listed in Table II. These propensities are calculated for all residue contacts that are at least 3 residues away in primary sequence. As expected, Cys-Cys has the highest propensities for contact interactions. Other residue pairs with the highest propensities for contact interactions $(P(i, j)=1.4-2.5)$ are pairs of hydrophobic residues (e.g., Met-Met, Ala-Ala, Ile-Ile, PhePhe, Ile-Leu, and Ile-Met). The group of residue 
pairs with the second highest propensities $(P(i, j)=$ $1.2-1.3)$ are ionizable residues with opposite charges (e.g., Arg-Asp, Arg-Glu, Asp-Lys, and Gly-Lys). Residue pairs with lowest alpha contact propensities $(P(i, j)=0.4-0.6)$ are dominated by pairs of ionizable residues of the same charge (e.g.,ArgLys, Asp-Glu, Lys-Lys, and Glu-Glu). The group of residue pairs with the second lowest alpha contact propensities $(P(i, j)=0.5-0.7)$ are between ionizable residues and hydrophobic residues (e.g., AspPhe, Asp-Ile, Asp-Leu, and Glu-Val). Noticeably, pairs of Pro and ionizable/polar residues also have very low propensity for contacting interactions, probably due to the lack of a backbone-NH for H-bonding interactions.

The confidence intervals of these propensity values given by the studentized bootstrap procedure indicate that most of them are estimated accurately. Among the 209 parameters excluding Cys-Cys interaction, the $95 \%$ confidence intervals for 153 contact propensities are $<0.2$, a very tight interval. The confidence intervals of 36 contact propensities are $<0.3$. Contact propensities with the largest confidence intervals around 0.6 are Trp-Trp, Met-Met, Cys-Met, and Cys-Trp.

Correlating and Clustering Similar Amino Acid Residues. The overall behavior of pairwise contact interactions for a specific residue type is determined by its 20 pairwise contact propensity values. These values represent a profile of contact interactions specific for the residue type, and can be represented as a 20 -dimensional vector $\boldsymbol{x}$.

We group the 20 types of amino acid residues by the Euclidean distance between the 20 vectors [34]. Fig 2 shows the grouping of the 20 amino acid residues obtained by hierarchical clustering. As an exploratory tool for data analysis, hierarchical clustering can discover interesting and informative grouping patterns in data [39]. In Fig 2, residues that have close contact propensity values to the 20 residue types are grouped together.

The pattern of groupings of residues clearly reflect the physical and geometric characteristics of the amino acid residues. Cys residue is different from all other residues, because of its propensity to form disulfide bond. The rest of the 19 residues can be broadly divided into two well defined branches of hydrophobic and hydrophilic residues. Among the hydrophilic residues, ionizable residues with positive charge and negative charge are grouped into two in-

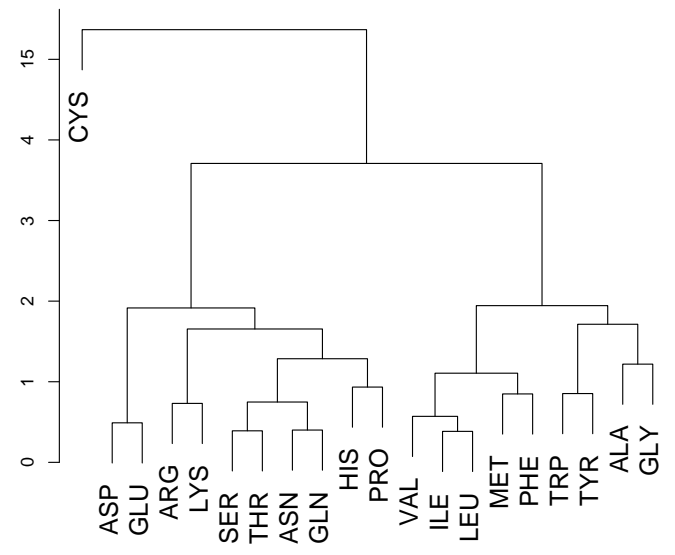

Figure 2: Grouping of the 20 types of amino acid residues by hierarchical clustering with complete linkage of the Euclidian distance between their 20-dimensional propensity vectors.

dividual small clusters. Hydroxyl-containing residues (Ser and Thr) and amide residues (Asn and Gln) are also clustered into two individual small clusters. These residues are all capable of forming side chain hydrogen bonds, and their clusters are neighboring with each other, forming a larger cluster of Ser, Thr, Asn, and Gln. Among the hydrophobic residues, the branched residues (Val, Ile, and Leu), and small residues (Ala and Gly) are grouped into their own clusters. Aromatic residues Trp and Tyr also group into one cluster. Phe has strong hydrophobicity and is group with other strongly hydrophobic amino acid residues, rather than clustering with the other two aromatic residues. Pro and His are grouped together, probably because both do not form strong favorable contacts with either hydrophilic or hydrophobic residues.

The clustering pattern of residues by alpha contact propensity also resembles to a certain extent the clustering pattern derived from mutation matrix BLOSUM50 [40] as reported by Murphy et al [41]. For example, Arg with Lys, Asn with Gln, Ser with Thr, and Glu with Asp are all clustered tightly with each other. In addition to the distinct grouping of Cys in our clustering result, a notable difference is that by alpha contact propensity residues Ser, Thr and Pro are grouped with hydrophobic residues instead of hydrophilic residues. 


\begin{tabular}{lcccc}
\hline \hline & Misfold & ifu & Asilomar & $\begin{array}{c}\text { pdberr } \\
\text { and spga }\end{array}$ \\
\hline RAPDF & $25 / 25$ & $32 / 44$ & $39 / 42$ & $5 / 5$ \\
CDF & $19 / 25$ & $20 / 44$ & $36 / 42$ & $5 / 5$ \\
GC & $25 / 25$ & $21 / 44$ & $32 / 42$ & $4 / 5$ \\
MJ & $25 / 25$ & $21 / 44$ & $34 / 42$ & $5 / 5$ \\
BT & $25 / 25$ & $22 / 44$ & $35 / 42$ & $4 / 5$ \\
Alpha & $25 / 25$ & $24 / 44$ & $37 / 42$ & $4 / 5$ \\
\hline \hline
\end{tabular}

Table II: Discriminating native structure from decoys in ProStAR data sets using alpha contact potential (Alpha). Results for each decoy subset are compared with those of other potentials, including RAPDF (Residue-specific Allatom Conditional Probability Discriminatory Function) [6], CDF (Contact Discriminatory Function) [6], MJ (Miyazawa \& Jernigan Potential) [5], BT (Betancourt \& Thirumalai Potential) [55] and GC (Geometry Center Based Potential). The first number in each cell is the number of correctly identified native proteins, and the second number is the total number of proteins in the subset. The first row lists the names of the decoy subsets. Data for RAPDF, and CDF are taken from Figure $1 \mathrm{~b}$ of reference [6].

Discriminating Native Structures from Decoys. An important method to determine the effectiveness of contact potential functions is to evaluate its success and failure in distinguish native protein structures from incorrectly folded decoy structures. We use three decoy data sets to assess alpha contact potential.

ProStar. The decoy sets in ProStar database [6] contain several subsets. The MISFOLD subset contains conformations of 25 sequences which are obtained by placing these sequences on structures of different folds with the same number of residues. The conformations of the side chains are obtained by Monte Carlo sampling [42]. Alpha contact potential succeeded in identifying all 25 native structures correctly (Table IIII).

For the AsILOMAR subset, alpha contact potential failed to identify 5 native structures out of 42 proteins. For the IFU subset, alpha contact potential failed for 20 out of 44 native structures. Alpha contact potential belongs to the class of residue-based potentials, similar to MJ, BT, CDF [6]. As pointed out in [7], decoys in IFU subset are especially challenging for residue-based potentials, because they are conformations of short loops, and have only a small number of residue contact interactions.

In the subset PDBERR and SGPA, the decoys are structures determined by diffraction but contain serious error, or are structures generated by molecu-
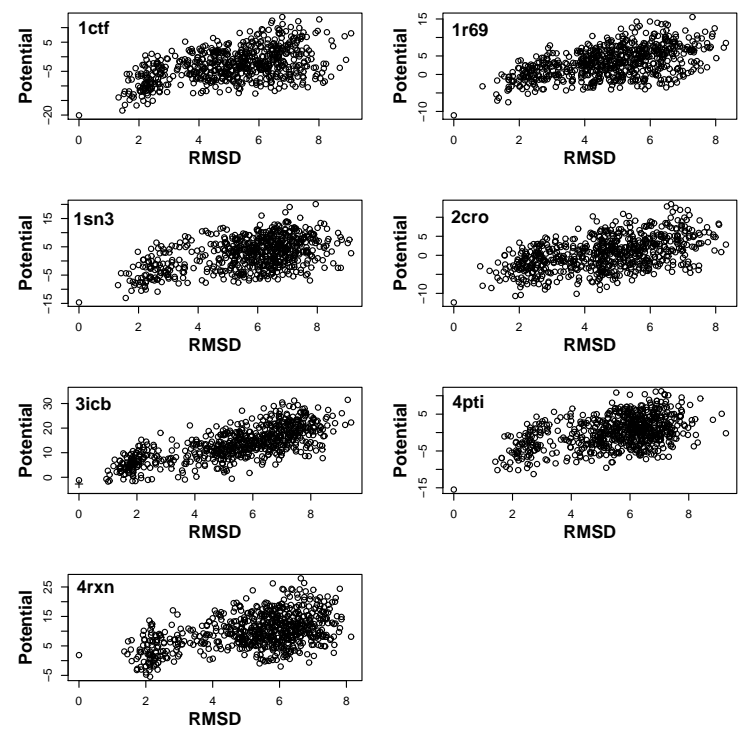

Figure 3: Energy evaluated by alpha contact potential plotted against the RMSD to native structures for conformations in Park\& Levitt Decoy Set. The alphabet of residues has 20 types of amino acids. For vitamin D-dependent calciumbinding protein (3icb, a structure with better resolution (4icb) has the lowest energy (denoted by " + ").

lar dynamics simulations starting from experimental conformations. Alpha contact potential missed one out of the five native structures.

Park 8 Levitt Set. This decoy test set contains native and near-native conformations of seven sequences, along with about 650 misfolded structures for each sequence. The positions of $C_{\alpha}$ of these decoys were generated by exhaustively enumerating ten selectively chosen residues in each protein using a 4state off-lattice model. All other residues were assigned the phi/psi value based on the best fit of a 4 -state model to the native chain. Conformations in the decoy sets all have low score by a variety of scoring functions, and have low RMSD to the native structure (Table IIIIII) [43].

The results of discrimination test are listed in Table IVIV and plotted in Fig 3. For five of the seven proteins, the native structures have lowest energy by alpha contact potential. For protein 3icb and 4rxn, the native structures have the 5th and 51st lowest energy values, respectively. For all proteins, decoys with the lowest energy are within $2.5 \AA$ RMSD to the native structure.

The protein $3 i \mathrm{cb}$ is a vitamin D-dependent calcium-binding protein. Although the energy of the 


\begin{tabular}{|c|c|c|c|c|c|}
\hline Decoy Set & Protein & Description & 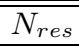 & 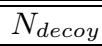 & cRMSD range \\
\hline \multirow{7}{*}{$\begin{array}{l}\text { 4state_ } \\
\text { reduced }\end{array}$} & $1 \mathrm{ctf}$ & C-terminal domain of the ribosomal protein L7/L12 & 68 & 630 & $2.16-10.16$ \\
\hline & 1 r69 & N-terminal domain of phage 434 repressor & 63 & 675 & $2.28-9.50$ \\
\hline & $1 \mathrm{sn} 3$ & Scorpion toxin variant 3 & 65 & 660 & $2.50-10.46$ \\
\hline & 2 cro & phage 434 Cro protein & 65 & 674 & $2.05-9.72$ \\
\hline & $3 \mathrm{icb}$ & Vitamin D-dependent calcium-binding protein & 75 & 653 & $1.81-10.74$ \\
\hline & 4 pti & trypsin inhibitor & 58 & 687 & $2.83-10.79$ \\
\hline & $4 \mathrm{rxn}$ & rubredoxin & 54 & 677 & $2.58-9.28$ \\
\hline \multirow{8}{*}{$\begin{array}{l}\text { lattice_ } \\
\text { ssfit }\end{array}$} & $1 \mathrm{beo}$ & $\beta$-Cryptogein & 98 & 2000 & $7.00-15.61$ \\
\hline & $1 \mathrm{ctf}$ & (see above) & 68 & 2000 & $5.45-12.81$ \\
\hline & $1 \mathrm{dkt}-\mathrm{A}$ & Human Cyclin Dependent Kinase Subunit, Type 1 & 72 & 2000 & $6.69-14.05$ \\
\hline & 1 fca & Ferredoxin & 55 & 2000 & $5.14-11.39$ \\
\hline & $1 \mathrm{nkl}$ & Nk-Lysin & 78 & 2000 & $5.27-13.64$ \\
\hline & $1 \mathrm{pgb}$ & $\begin{array}{l}\text { B1 immunoglobulin-binding domain of } \\
\text { streptococcal protein } \mathrm{G}\end{array}$ & 56 & 2000 & $5.81-12.91$ \\
\hline & 1 trl-A & $\begin{array}{l}\text { NMR solution structure of the C-terminal fragment } \\
255-316 \text { of thermolysin }\end{array}$ & 62 & 2000 & $5.38-12.52$ \\
\hline & $4 \mathrm{icb}$ & Calcium-Binding Protein & 76 & 2000 & $4.74-12.92$ \\
\hline \multirow[t]{10}{*}{ lmds } & 1b0n-B & Sini protein subunit & 39 & 497 & $2.45-6.03$ \\
\hline & $1 \mathrm{bba}$ & Pancreatic hormone (AVE. NMR) & 36 & 500 & $2.78-8.91$ \\
\hline & $1 \mathrm{ctf}$ & (see above) & 68 & 497 & $3.59-12.53$ \\
\hline & $1 \mathrm{dtk}$ & Dendrotoxin K (NMR) & 57 & 215 & $4.32-12.58$ \\
\hline & $1 \mathrm{fc} 2-\mathrm{C}$ & $\begin{array}{l}\text { Fragment B of protein A (Complexed to } \\
\text { immunoglobin Fc) }\end{array}$ & 43 & 500 & $4.00-8.45$ \\
\hline & 1igd & 3rd IgG-binding domain from streptococcal protein G & 61 & 500 & $3.11-12.56$ \\
\hline & 1shf-A & $\begin{array}{l}\text { Fyn Proto-Oncogene Tyrosine } \\
\text { Kinase subunit (SH3 domain) }\end{array}$ & 59 & 437 & $4.39-12.35$ \\
\hline & $2 \mathrm{cro}$ & (see above) & 65 & 500 & $3.87-13.48$ \\
\hline & 2ovo & 3rd domain of silver pheasant ovomucoid & 56 & 347 & $4.38-13.38$ \\
\hline & 4 pti & (see above) & 58 & 343 & $4.94-13.18$ \\
\hline
\end{tabular}

Table III: Description of proteins in the 4-STATE-REDUCED decoy set, LATTICE-SSFIT decoy set and LMDS decoy set. The number of decoy structures and the CRMSD ranges are listed.

native structure ranks as the 5th lowest energy, the RMSDs of the first four lowest energy decoys are all within $2.0 \AA$ RMSD to the native structure. For a higher resolution structure (4icb at $1.60 \AA$ ) of this protein, the energy of $4 i \mathrm{cb}$ by alpha contact potential is lower than any of the decoys. It is possible that this mis-classification might be due to the lower resolution of structure of $3 \mathrm{icb}$.

Rubredoxin (4rxn) is an iron-sulfur protein. A $\mathrm{Fe}(\mathrm{III})$ ion is covalently bound in this structure with four Cys sulfur atoms, preventing these four Cys from forming two possible disulfide bonds. Because the protein description and the contact potential do not contain any information about the important covalent bonds of Cys with Fe-S cluster, it is reasonable to expect that native structure will not be of lowest energy if these important covalent bond interactions are unaccounted for. It is possible that the structure of rubredoxin might be different without the Fe$\mathrm{S}$ cluster. The decoys at the lowest energy states form one or two fictitious disulfide bridges, and all are near-native structures with RMSD around $2 \AA$ to the native structure. MJ and BT potential work better on 4rxn because they classify the four Cys-Cys contacts as disulfide bonds.

Lattice_ssfit Set. The LATTICE_SSFIT set contains conformations for eight small proteins generated by $a b$ initio protein structure prediction methods [44, 45]. The conformational space of a sequence was exhaustively enumerated on a tetrahedral lattice. A lattice-based scoring function was used to select the 10,000 best scoring conformations. Secondary struc- 


\begin{tabular}{|c|c|c|c|c|c|c|c|c|c|c|c|}
\hline \multirow[b]{2}{*}{ Decoy set } & \multirow[b]{2}{*}{ PDB } & \multicolumn{4}{|c|}{$\overline{\overline{H_{\alpha}}}$} & \multicolumn{2}{|c|}{$\overline{\overline{H_{g c}}}$} & \multicolumn{2}{|c|}{$\overline{\overline{H_{M J}}}$} & \multicolumn{2}{|c|}{$\overline{H_{B T}}$} \\
\hline & & ${ }^{a}$ Rank & ${ }^{b} Z$ & ${ }^{c} \bar{r}$ & ${ }^{d} f / 1,000$ & Rank & $Z$ & Rank & $Z$ & Rank & $Z$ \\
\hline \multirow{7}{*}{$\begin{array}{l}\text { sstate_ }_{-} \\
\text {reduced }\end{array}$} & $1 \mathrm{ctf}$ & 1 & 3.08 & 1.0 & 1,000 & 1 & 3.42 & 1 & 3.73 & 1 & 3.86 \\
\hline & 1r69 & 1 & 3.33 & 1.0 & 1,000 & 8 & 2.34 & 1 & 4.11 & 1 & 4.47 \\
\hline & $1 \mathrm{sn} 3$ & 1 & 3.10 & 1.0 & 1,000 & 8 & 2.49 & 2 & 3.17 & 6 & 2.97 \\
\hline & $2 \mathrm{cro}$ & 1 & 3.00 & 1.0 & 1,000 & 2 & 2.91 & 1 & 4.29 & 1 & 3.92 \\
\hline & $3 \mathrm{icb}$ & 5 & 2.19 & 3.4 & 52 & 10 & 2.14 & 2 & 2.80 & 1 & 2.83 \\
\hline & $4 p t i$ & 1 & 2.30 & 1.0 & 1,000 & 11 & 2.28 & 3 & 3.16 & 5 & 2.65 \\
\hline & $4 r x n$ & 51 & 1.22 & 43.0 & 0 & 1 & 2.75 & 1 & 3.09 & 1 & 3.01 \\
\hline \multirow{8}{*}{$\begin{array}{l}\text { lattice_ } \\
\text { ssfit }\end{array}$} & 1beo & 1 & 4.74 & 1.1 & 923 & 2 & 3.69 & 1 & 4.74 & 1 & 7.29 \\
\hline & $1 \mathrm{ctf}$ & 1 & 4.62 & 1.0 & 1,000 & 1 & 5.09 & 1 & 5.35 & 1 & 6.99 \\
\hline & $1 \mathrm{dkt}-\mathrm{A}$ & 1 & 4.33 & 1.0 & 1,000 & 15 & 2.38 & 32 & 2.41 & 5 & 3.49 \\
\hline & $1 \mathrm{fca}$ & 40 & 2.01 & 32.0 & 0 & 254 & 1.18 & 5 & 3.40 & 2 & $3.92^{\circ} \mathrm{q}$ \\
\hline & $1 \mathrm{nkl}$ & 1 & 5.21 & 1.0 & 1,000 & 1 & 7.20 & 1 & 5.09 & 1 & 7.28 \\
\hline & $1 \mathrm{pgb}$ & 1 & 3.31 & 1.0 & 964 & 32 & 2.18 & 3 & 3.78 & 2 & 3.82 \\
\hline & 1 trl-A & 5 & 3.35 & 6.2 & 0 & 504 & 0.63 & 4 & 2.91 & 2 & 3.82 \\
\hline & $4 \mathrm{icb}$ & 1 & 4.59 & 1.0 & 1,000 & 1 & 4.11 & 1 & 3.67 & 1 & 5.07 \\
\hline \multirow[t]{10}{*}{ lmds } & 1b0n-B & 2 & 3.13 & 1.5 & 525 & 99 & 0.85 & 1 & 2.65 & 2 & 2.50 \\
\hline & $1 \mathrm{bba}$ & 217 & 0.03 & 340.8 & 0 & 441 & -1.11 & 364 & -0.64 & 234 & 0.04 \\
\hline & $1 \mathrm{ctf}$ & 1 & 3.12 & 1.0 & 1,000 & 74 & 1.09 & 1 & 3.86 & 1 & 3.15 \\
\hline & $1 \mathrm{dtk}$ & 2 & 2.13 & 2.2 & 234 & 173 & -0.92 & 13 & 1.71 & 122 & -0.08 \\
\hline & $1 \mathrm{fc} 2-\mathrm{C}$ & 500 & -3.68 & 500 & 0 & 480 & -1.63 & 501 & -6.24 & 501 & -5.11 \\
\hline & 1igd & 9 & 2.43 & 14.0 & 0 & 138 & 0.61 & 1 & 3.25 & 1 & 3.76 \\
\hline & 1 shf-A & 17 & 1.46 & 8.2 & 0 & 322 & -0.57 & 11 & 1.30 & 16 & 1.06 \\
\hline & $2 \mathrm{cro}$ & 1 & 4.36 & 1.0 & 999 & 159 & 0.44 & 1 & 5.07 & 1 & 4.01 \\
\hline & 2ovo & 3 & 3.07 & 5.2 & 29 & 326 & -1.34 & 2 & 3.25 & 31 & 1.29 \\
\hline & $4 p t i$ & 9 & 2.23 & 7.0 & 0 & 242 & -0.49 & 4 & 2.53 & 117 & 0.42 \\
\hline
\end{tabular}

Table IV: Discriminating native structures using alpha contact potential $H_{\alpha}$ and potential by cut-off distance between geometric centers of side chains $H_{g c}$. The 4-state-reduced, LATTICE-SSFIT, and LMDS decoy sets are tested.

${ }^{a}$ Rank of native structures.

${ }^{b} z=\bar{E}-E_{\text {native }} / \sigma ; \bar{E}$ and $\sigma$ are the mean and standard deviation of the energy values of conformations, respectively.

${ }^{c}$ Average ranking of native structures in energy evaluated using 1,000 bootstrapped potential values;

$d$ The number of times of a native structure is ranked to have the lowest energy.

tures were fitted to these conformations using a 4state model [43]. The 10,000 conformations were further scored using a combination of an all-atom scoring function [6], a hydrophobic compactness function, and a one-point per residue scoring function [46]. The 2,000 best scoring conformations for each protein are selected as decoys for this data set.

The result (Table IVIV) shows that for six out of eight proteins, no decoy structures score better than the native structure. The exceptions are $1 \mathrm{fca}$ and 1trl. Similar to $4 \mathrm{rxn}$ in the Park \& Levitt decoy set, ferrodoxin $1 \mathrm{fca}$ contains a Fe-S cluster. Its four Cys residues form four covalent bond with the four $\mathrm{Fe}(\mathrm{III})$ irons, instead of two disulfide bridges. These critical contacts again are unaccounted for in the alpha contact potential, and therefore the native structure of this protein was not identified successfully.
$1 \mathrm{trla}$ is a NMR solution structure of the C-terminal fragment (255-316) of thermolysin. NMR structures are far more difficult to recognize, as discussed in detail in [10]. They are usually represented as an ensemble of conformations. The contact energies of conformations in the ensemble can be substantially different. It is conceivable that an energy function valid for crystal structures cannot reliably recognize native NMR structures [10]. In addition, the structure $1 \mathrm{trla}$ occurs in a dimeric state in the original PDB file. There is substantial interaction between the two chains. Because of this, it is unclear whether single subunit of $1 \mathrm{trla}$ in monomeric state would retain the same conformation.

The decoy structures in this data set are generated by ab initio methods. None of them are near-native, and all have cRMSD to the native structure greater 
than $4.7 \AA$ (Table IVIII). When decoys are so different from the native conformation, energy evaluated using alpha contact potential shows little correlation with the RMSD. The lowest energy decoys in this data set all have large RMSD, similar to results reported in [47] (data not shown).

Lmds Set. The local minima decoy set (LMDS) contains decoys that were derived from the experimentally obtained secondary structures of ten small proteins that belong to diverse structural classes. Each decoy is a local minimum of a "hand made" energy function [48-51]. Ten thousand initial conformations were generated for each protein by randomizing the torsion angles of the loop regions [52]. The adjacent local minima were found by truncated NewtonRaphson minimization in torsion space. Each protein is represented in the decoy set by its 500 lowest energy local minima.

The alpha contact energy function works fairly well in the recognition of $1 \mathrm{~b} 0 \mathrm{n}-\mathrm{B}, 1 \mathrm{ctf}, 1 \mathrm{dtk}, 2 \mathrm{cro}$ and 2ovo. 1dtk (Dendrotoxin K) contains three disulfide bonds in its native structure. However, in most of its 215 decoys, six Cys residues are spatially arranged together and form on average seven Cys-Cys contacts. For some decoys, up to 15 CysCys contacts by distance cut-off can be found. The ability of discriminating disulfide bonded versus nondisulfide bonded Cys-Cys contacts probably makes the alpha contact potential discriminate better than the MJ and the BT potentials. The native structure of 1igd (IgG-binding domain from streptococcal protein $\mathrm{G}$ ) ranks first by the MJ and the BT potential, but ranks 9 th by alpha contact potential. The recognition of $1 \mathrm{bba}$ and $1 \mathrm{fc} 2-\mathrm{C}$ in this set failed for all residue-based contact potentials. $1 \mathrm{bba}$ is an atypical structure of a small protein determined by NMR, which forms a helix with random coil. $1 \mathrm{fc} 2-\mathrm{C}$ is a fragment of protein complexed with an immunoglobulin molecule. It possible that this protein may not maintain the same conformation without the complexed immunoglobulin.

By the criterion of the ranking of native protein, with the exception of the ion-sulfur proteins 4 rxn and $1 \mathrm{fca}$, the overall results shown in Table IVII and Table IVIV indicates that the performance of alpha contact potential in discriminating native protein from decoys is better than that of MJ and BT potentials for the MISFOLD, IFU, ASILOMAR, 4STATE_REDUCED sets and the LATTICE_SSFIT set, and has comparable results for the LMDS set, the PDBERR set, and the SPGA set.

\section{Discussion}

Contact Definition. The alpha contacts introduced in this work are different from contacts by cutoff distances. Atoms in alpha contacts are all within a distance that depends on the identities of the two atoms. In this study, this distance is equal to the sum of the van der Waals radii of the two atoms, plus $2 \times 0.5 \AA$. Unlike contacts by distance cut-off, this distance is not a single fixed constant but depends on the atom types. Another important distinction of alpha contact is that only a subset of atoms satisfying the distance criterion will be counted as physical nearest neighbors, because we have an additional criterion: contacting atoms must have intersecting Voronoi cells. Alpha contacts represents the geometry more accurately and can capture contact interactions due to side chain size and orientation [25]. In addition, no fictitious contacts will be introduced between two atoms when there is a third intervening atom [24]. Perhaps this is the reason why alpha contact potential is sensitive to the presence of Fe-S clusters and other hetero atoms, which can be potentially exploited for determining whether a protein structure should contain hetero atoms.

For the 1,045 proteins in the PDBSELECT dataset, we compare contacts identified by distance cut-off with the threshold of two van der Waals atom radii plus $2 \times 0.5 \AA$ and contacts identified by the alpha shape. We found that about $30-50 \%$ of atom contacts detected by distance cutoffs are blocked by a third atom and hence do not have physical interactions. As a result, $3-6 \%$ residue contacts detected by distance cutoffs do not interact physically. Inclusion of these fictitious contact is problematic, especially in developing all-atom contact potentials, as well as in future studies when higher order interactions in the form of three or four body contacts are incorporated.

Evaluating Discrimination of Alpha Contact Propensities by Bootstrap. How robust is the results of decoy discrimination to the specific values of alpha contact potentials and the specific choices of the structures in the database? We further make use of the bootstrap resampling technique to evaluate the reliability of the discrimination results. As discussed earlier, we resampled 1,045 proteins in PDBSELECT independently $R=1,000$ times with replacement allowed, and obtain 1,000 contact propensity matrices. Each is then used to discriminate the decoys in Table III. We use two parameters: $\bar{r}$, the aver- 
age ranking of the native structure, and $f$, the times a native structure ranked as the structure with the lowest energy. Table IV shows that for many decoy sets (e.g., 1ctf, 1r69, 1sn3, 2cro, and 4pti in the 4STATE_REDUCED set), the native structure always ranks first in the 1,000 bootstrapped energy evaluations. The performance using 1,000 different sets of "bootstrapped" potential values validates the robustness of the method deriving the alpha contact potential and the informativeness of the underlying protein structure database.

\section{Comparison with Contact by Geometric Cen-} ters. Contact definition by distance cut-off is widely used in the development of many potential functions. Here we compare potentials obtained by alpha contact, denoted as $H_{\alpha}$, and by contact defined by cut-off distance between geometric centers of side chains, denoted as $H_{g c}$. Following reference [53], two residues are declared to be in contact if the distance $d$ between the geometric centers of their side chains is: $2 \AA<d<6.5 \AA$. Geometric center based contact potential $H_{g c}$ are developed using the same PDBSELECT data, with the same null model as that of alpha contact potential, and similarly counting only contact between residues that are three or more residues apart. Therefore, any difference between $H_{\alpha}$ and $H_{g c}$ is solely due to different geometric representation.

The log values of the parameters of $H_{\alpha}$ and $H_{g c}$ have an overall correlation coefficient of $\rho=0.77$. However, the contact maps of individual proteins by these two different contact definition are often substantially different. As an example, alpha shape dual simplicial complex gives significantly more contacts than cut-off distance by geometric centers of side chain for protein 1abe (Fig 4).

Fig 5 illustrates why such a discrepancy exists between these two contact models (see Figure 5 legend). The strong correlation between $H_{\alpha}$ and $H_{g c}$ is deceptive and this is reflected in another aspect. Although the correlation coefficient is high, the pairwise contact potentials may have very different values for $H_{\alpha}$ and $H_{g c} . H_{g c}$ categorically gives much higher propensity values for interactions between small residues. For example, $H_{g c}$ for Gly-Gly and Ala-Gly are 4.55 and 3.04, respectively, but $H_{\alpha}$ are only 1.48 and 1.39, respectively. Gly-Pro interaction is strongly favorable by $H_{g c}(P(G l y$, Pro $)=1.84)$, but is unfavorable by $H_{\alpha}(0.87)$. On the other hand, $H_{g c}$ gives much lower propensity values for interactions between large residues. For example, $H_{g c}$ for Trp-Trp and Phe-Trp

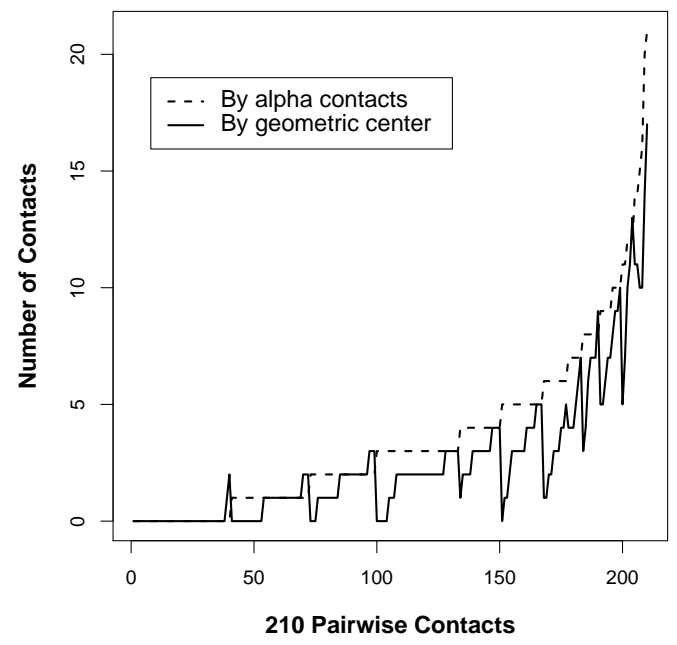

Figure 4: Difference in contact histograms between the two contact definitions of alpha contact and contact by geometric centers for protein structure $1 \mathrm{abe}$. Data along $x$-axis is arranged in ascending order by the frequency of contact pairs in the alpha shape contact model. There are frequently significant less contacts when contact defined by distance between geometric centers is used.

are 0.39 and 0.56 , respectively, but $H_{\alpha}$ are 1.75 and 1.65 , respectively. In addition, many pair contact interactions between Trp and another residue with large side chains (such as Arg, Tyr, Phe, His, Leu, Ile, and Met) are unfavorable $(<1.0)$ by $H_{g c}$, but are favorable by $H_{\alpha}$.

These differences lead to different discrimination in identifying native and near-native protein structures from decoy structures. Because it is impossible to define refined potential for Cys-Cys contacts in $H_{g c}$ model as in alpha contact model, we exclude proteins containing Cys-Cys contacts to avoid complication for comparison. Table IVIV shows that $H_{g c}$ can only recognize two native structures out of nine proteins in the union set of the 4-state decoy set and the lattice-ssfit decoy set. In addition, the $z$ scores for native structures are generally higher when using $H_{\alpha}$ than $H_{g c}$. For the 4-state decoy set, the correlation coefficient $\rho$ by $H_{\alpha}$ is also higher. These results indicate that geometric description of protein structures are important, and contact model by alpha shape are more accurate with more discriminative information for identifying native-like structures. However, the cut-off distance approach may be more convenient to implement, and it is possible to gain further improvement by setting the values of the cut-off threshold as a variable depending on the type of contacting residue 


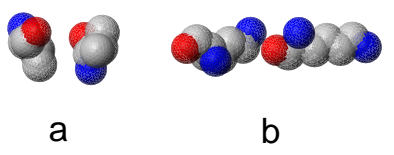

Figure 5: Alpha contact maps provide accurate geometric descriptions. a) Two Val residues are nearly parallel to each other. Their geometric centers are close enough, but no physical contacts occur between them. b) Two Lys are oriented linearly in opposite direction. Their geometric centers are far away from each other, but a hydrogen bond forms between $\mathrm{O}$ from one Lys and $\mathrm{N}$ from the other Lys. Alpha contact definition correctly identify a) as "in contact" and b) as not "in contact". Contact model by distance cut-off of geometric centers of side chains give different contact assignment in both cases.

pairs.

Comparison with Other Potentials. We further compare alpha contact potential with several previously developed residue contact potentials, including MJ [54], BT [55], SK [33], and TD [9] potentials. We take the values of MJ potential from Table 3 of reference [54]. Following the author's recommendation, the average hydrophobicity $\left(e_{r r}=-\right.$ 2.55) was subtracted from the potential, and a pair of residues is declared to be in contact if the geometric centers of their side chain is within an interval of $2.0 \AA$ to $6.5 \AA$. The values of BT potential is taken from [55] and was obtained by rescaling MJ potentials with Thr as the reference solvent [55]. The correlation coefficients $\rho$ and dispersions [55] between each pair of potentials are shown in table Table VV. The correlation coefficients $\rho$ for alpha contact potential $\log P(i, j)$ and these residue contact potentials are: $\rho=0.66,0.80,0.61$, and 0.66 for MJ, BT, SK, and TD potentials, respectively. The dispersion as defined in [55] (p.363, Formula 4) are 1.45, 0.28, 0.51, and 0.39 , respectively. Because the contact map obtained by alpha edges can be substantially different from other contact definition (Fig 4), the absolute value of energy by alpha contact potential and by other potentials for the same structure can be substantially different.
Long Range Interactions. Interactions between residues with large sequence distance $d$ are relatively rare. We found that they occur more likely in the interior of a protein than on the surface (data not shown). Identifying such interactions are of particular interest, because these interactions result in significant reduction of conformational entropy. Prediction of protein structures seem to be most difficult for those with large contact order [56], namely, those with significant amount of interactions between residues with large sequence distances.

The bootstrap procedure introduced here provides a method to reliably identify the contact pairs of long sequence separation whose propensity values can be confidently assessed (see Supplementary Online Material for tables of alpha contact potential for $d \geq 30$ ). Among all possible 210 pairwise interactions, 9 contact pairs with high propensity (lower value of $95 \%$ confidence interval $>1.5$ ) can be reliably assessed for $d>30$. In addition to Cys-Cys, they include hydrophobic-hydrophobic interactions (GlyGly, Met-Met, Ile-Ile, Phe-Phe, Val-Val, Met-Phe), salt-bridge interactions (Arg-Asp, Asp-Lys), and ProTrp.

Some long range interactions are clearly associated with specific secondary structures. After correction for prior probability to be in a particular secondary structure, we found that Met-Met contact has a high propensity to occur between two helices $(h)$ or two beta-strands $(s)\left(P(\text { Met }, \text { Met })_{h h}=\right.$ $\left.2.4, P(\text { Met, Met })_{s s}=2.3\right)$, and a low propensity to occur between either a helix and a coil (c) $\left(P(\text { Met }, \text { Met })_{h c}=0.65\right)$, or a strand and a coil $\left(P(\text { Met }, \text { Met })_{s c}=0.56\right)$. Similarly, because Gly is a helix breaker, long-range Gly-Gly contact has a high propensity to occur between two coils $\left(P(G l y, G l y)_{c c}=3.2\right)$, and low propensity to occur between two helices $\left(P(G l y, G l y)_{h h}=0.53\right)$.

Reduced Alphabet for Amino Acid Residues. The clustering of pairwise alpha contact potentials shown in Figure 2 suggests that many residues behave similarly in contact interactions. This points to possible degeneracy of the amino acid alphabet $[16,17]$. Reduced alphabet is important because a smaller size of alphabet will lead to exponentially more efficient sampling methods in sequence design and protein engineering [57-61]. Many experimental and computational studies already suggested that a minimum number of amino acid residue types far less than 20 may be adequate for protein folding $[40,62$ 


\begin{tabular}{l|cccccc}
\hline \hline & Alpha & MJ & BT & GC & SK & TD \\
\hline Alpha & $1 / 0$ & $0.66 / 1.45$ & $0.80 / 0.28$ & $0.77 / 0.41$ & $0.61 / 0.51$ & $0.66 / 0.39$ \\
MJ & $0.66 / 1.45$ & $1 / 0$ & $0.66 / 1.43$ & $0.37 / 1.60$ & $0.73 / 1.29$ & $0.67 / 1.35$ \\
BT & $0.80 / 0.25$ & $0.66 / 1.43$ & $1 / 0$ & $0.49 / 0.56$ & $0.76 / 0.41$ & $0.63 / 0.37$ \\
GC & $0.77 / 0.41$ & $0.37 / 1.60$ & $0.49 / 0.56$ & $1 / 0$ & $0.15 / 0.81$ & $0.43 / 0.63$ \\
SK & $0.61 / 0.55$ & $0.76 / 1.29$ & $0.82 / 0.41$ & $0.15 / 0.81$ & $1 / 0$ & $0.64 / 0.52$ \\
TD & $0.66 / 0.39$ & $0.67 / 1.35$ & $0.63 / 0.37$ & $0.43 / 0.63$ & $0.64 / 0.52$ & $1 / 0$ \\
\hline \hline
\end{tabular}

Table V: The correlation coefficients and dispersions [55] between each pair of potentials. The first number of each cell is the correlation coefficient between each pair of potentials. The second number is the dispersion between each pair of potentials.

64]. Wang and Wang examined different ways to reduced MJ interaction matrix and concluded that by minimizing mismatches, a reduced alphabet of just five amino acid residue types can be used to construct sequences with good foldability and kinetic accessibility [65]. The reduced 5 alphabet set coincide with the same alphabet set reported in the work of Riddle et al, where fully functional constructs for a small 57-residue beta-barrel protein can be experimentally obtained when residues in 38 out of 40 selected amino residues are drawn from the alphabet set of I, K, E, A, and G. Murphy et al further examined reduced alphabets based on BLOSUM50 substitution matrix [41]. When using a variety of reduced alphabets with size ranging from 10 to 20, they found that there is little loss of the information necessary to select structural homologs in a database of representative protein sequences using dynamic programming based global alignment.

We continue investigation in this direction and study the capability of various reduced alphabet sets in discriminating native proteins from decoys. Fig 2 provides a natural way of reducing the residue alphabet set that is similar to the approach used by Murphy et al [41]. By placing a horizontal line at different vertical heights, we can obtain a reduced residue alphabet that is determined by the heights of the branching points in the dendrogram from the hierarchical clustering. For example, we can have an alphabet of 7 residue types at height about 1.5: $\mathcal{A}=\{\mathrm{D}, \mathrm{E}\}, \mathcal{B}=\{\mathrm{R}, \mathrm{K}\}, \mathcal{C}=\{\mathrm{S}, \mathrm{T}, \mathrm{N}, \mathrm{Q}, \mathrm{H}, \mathrm{P}\}$, $\mathcal{D}=\{\mathrm{V}, \mathrm{I}, \mathrm{L}, \mathrm{M}, \mathrm{F}, \mathrm{W}\}, \mathcal{E}=\{\mathrm{W}, \mathrm{Y}\}, \mathcal{F}=\{\mathrm{A}$, $\mathrm{G}\}$, and $\mathcal{G}=\{\mathrm{C}\}$. An alphabet of two residue types would take Cys as a residue type, and everything else grouped into another residue type. An alphabet of three residue types would have Cys, polar residues, and hydrophobic residues.

Does reduced alphabet still capture the basic in-
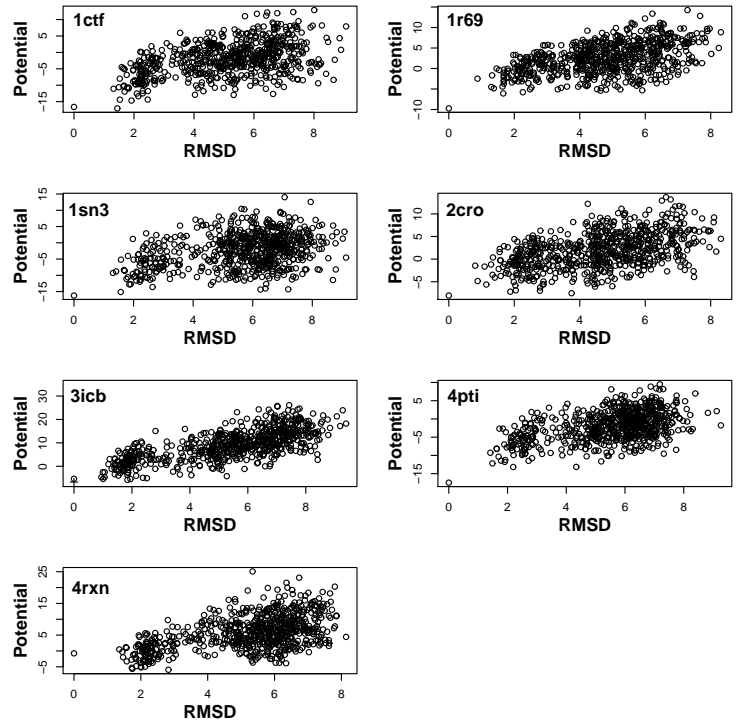

Figure 6: Energy evaluated by alpha contact potential plotted against the RMSD to native structures for conformations in Park\& Levitt Decoy Set. The alphabet of residues has 9 types of amino acids. The discrimination is similar to that shown in Fig 3. 4icb is denoted by "+" and has the lowest energy.

formation of protein contact interactions? We use Equation 1 to estimate pairwise alpha contact potentials for reduced alphabet size of $2,3,5,7,8,9,10$, 11,15 , and 20 , and test their effectiveness in selecting native structure from decoys in the Park and Levitt data set. Fig 6 shows the result when an alphabet set of 9 residue types, plus the three types of Cys-Cys contacts are used. Remarkably, the discrimination of native conformation from decoys is almost as good as when there are 20 different residue types.

Figure 7 shows the detailed results of discriminating native structure of $3 i \mathrm{cb}$ from decoys using poten- 


\begin{tabular}{l|ccccccc}
\hline \hline${ }^{*}|\Sigma|$ & 1ctf & 1r69 & 1sn3 & 2cro & 3icb & 4pti & 4rxn \\
\hline 2 & 0.31 & 0.35 & 0.33 & -0.15 & 0.13 & 0.40 & 0.17 \\
3 & 0.39 & 0.44 & 0.48 & 0.34 & 0.73 & 0.44 & 0.39 \\
5 & 0.41 & 0.47 & 0.47 & 0.38 & 0.71 & 0.47 & 0.51 \\
7 & 0.45 & 0.44 & 0.48 & 0.46 & 0.71 & 0.49 & 0.48 \\
8 & 0.54 & 0.46 & 0.42 & 0.48 & 0.68 & 0.52 & 0.50 \\
9 & 0.47 & 0.48 & 0.47 & 0.47 & 0.72 & 0.47 & 0.49 \\
10 & 0.47 & 0.49 & 0.47 & 0.48 & 0.71 & 0.48 & 0.51 \\
11 & 0.47 & 0.50 & 0.49 & 0.47 & 0.72 & 0.49 & 0.51 \\
15 & 0.49 & 0.50 & 0.49 & 0.50 & 0.71 & 0.47 & 0.49 \\
20 & 0.49 & 0.52 & 0.49 & 0.50 & 0.74 & 0.46 & 0.53 \\
\hline \hline
\end{tabular}

Table VI: Correlation coefficients $\rho$ between energy evaluated using alpha contact potential and RMSD to the native structures for decoys in the Park and Levitt Set. Alpha contact potentials with alphabet containing different number of residue classes are used.

${ }^{*}|\Sigma|$ denotes the number of residue classes in the alphabet $\Sigma$ as obtained from Fig 2.

tials derived from different alphabet sets. The average RMSDs of $n$ decoys with lowest energy by potentials of different alphabets are calculated. Smaller average RMSD of these lowest energy decoys indicates that a large fraction of them are near-native structures. This would suggest good discrimination. The top $n=100$ decoys of lowest energy are all found to have very similar average RMSD to the native structure, regardless the size of the alphabet. This suggests that alphabet with just a few residues have respectable results in decoy discrimination. Table VIVI further shows the correlation coefficient of energy and RMSD for all proteins in the Park and Levitt data set when using different alphabets. The results indicate that an alphabet of 7 residues would have very similar performance as an alphabet with 20 residues in discriminating decoys. Our results extended earlier work where subjectively defined alphabet sets were used to extract contact residue potentials by an iterative optimization method [9]. It was found that potential derived using such reduced alphabets were effective in discriminating decoys generated by gapless threading. Here we showed that similar conclusion can be drawn for statistical potential using alphabet sets derived from natural clustering of residues, in discriminating natives against more stringent compact decoy conformations generated by off-lattice model [43]. Our conclusion is also consistent with a recent study where it is shown that much of the information in pairwise contact potential is related to just a few variables such as hydropathy, charge, disulfide bonding, and residue burial [66].

Although the alphabets we used have different number of residues, they are all developed with one aspect in common, i.e., the contacts are all derived from the dual simplicial complexes, which provides a faithful representation of the geometry. This suggests that as long as the same space filling pattern is conserved, the specific residue types are not critical in many cases. It seems that packing geometry plays a very important role, but the specific residue types are often replaceable. This observation is consistent with experimental results where it is well known that proteins are robust against many mutations.

Edge Simplices and Tetrahedron Simplices. Pairwise alpha contact potential only considers the edge simplices or 1-simplices in the dual simplicial complex. There have been several studies of statistical potential based on 3-simplices or tetrahedra [67-69]. In the work of Tropsha et al, 3-simplices are obtained from unweighted Delaunay triangulation $[67,68]$. In these studies, all residues are treated as balls of equal size located at $\mathrm{C}_{\alpha}$ or $\mathrm{C}_{\beta}$ positions, and a cut-off distance is used to remove tetrahedra that are considered to be too large. Our approach is different. Our model is instead based on the weighted dual simplicial complex, a different simplicial complex formed by a subset of the simplices from the weighted Delaunay triangulation of all atoms in the molecule. The dual simplicial complex or the alpha shape allows modeling at atomic level. Therefore, in our approach contacts can be defined by the full side chain and main chain atoms. Additionally, atoms are assigned with appropriate non-uniform van der Waals radii [31]. Finally, because the dual simplicial complex reflects the precise contact geometry, we avoid the use of heuristic cut-off thresholds necessary 


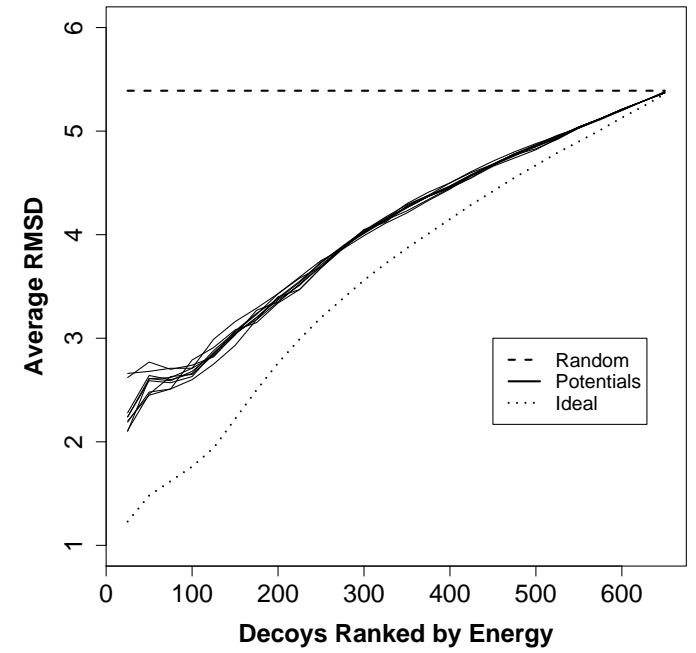

Figure 7: Discriminating native structure of 3icb from decoys using different alphabets. For each alphabet, we rank the decoys by their energy. The average RMSD to the native structure is then calculated for the top $n$ decoys at different $n$ values. Top dashed line represents the average RMSD if the decoys are chosen randomly; Dotted line represents the ideal case that $n$ decoys with smallest RMSD are chosen by an ideal function; The other lines represent the average RMSD of decoys chosen by potentials of an alphabet with 2-20 amino acid types.

to eliminate a subset of the simplices from the Delaunay triangulation. Our contacts represents accurately geometry of the structure. We discussed earlier the differences between the alpha contact and contact by distance cut-off between geometric centers of side chains. It is conceivable that similar difference will result between alpha contact and the approach described in $[67,68]$.

Summary. In this work, we introduced a novel representation of protein structures using edge simplices of the alpha shape, or the dual simplicial complex of the protein structure. By describing pairwise contact interactions with simplicial edges, we developed alpha contact potential based on the statistics of edge simplices. We also developed a bootstrap model which provides confidence interval estimations, including those of long range interactions. We found that alpha contact potential performs well in decoy structure discrimination. By comparing with alternative contact potential, we conclude that geometric representation of contact interaction is important, but the specific residue types are often interchangeable.

\section{Acknowledgment}

We thank Dr. Hui Lu for very helpful discussion. This work is supported by funding from National Science Foundation CAREER DBI0133856, DBI0078270 and MCB998008 and American Chemical Society/Petroleum Research Fund 35616-G7.

\section{References}

[1] J. Skolnick and A. Kolinski. Monte carlo methods in chemical physics. Advances in Chemical Physics, 105:203-242, 1999.

[2] W.L. Jorgensen and J. Tirado-Rives. The OPLS potential function for proteins: Energy minimizations for crystals of cyclic peptides and crambin. J. Amer. Chem. Soc., 110:1657-1666, 1988.

[3] T. Lazaridis and M. Karplus. Discrimination of the native from misfolded protein models with an energy function including implicit solvation. J. Mol. Biol., 288:477-487, 1999.

[4] D.W. Gatchell, S. Dennis, and S. Vajda. Discrimination of near-native protein structures from misfolded models by empirical free energy functions. Proteins, 41:518-534, 2000.

[5] S. Miyazawa and R. Jernigan. Estimation of effective interresidue contact energies from protein crystal structures: quasi-chemical approximation. Macromolecules, 18:534-552, 1985.

[6] R. Samudrala and J. Moult. An all-atom distance-dependent conditional probability discriminatory function for protein structure prediction. J. Mol. Biol., 275:895-916, 1998.

[7] H. Lu and J. Skolnick. A distance-dependent atomic knowledge-based potential for improved protein structure selection. Proteins, 44:223$232,2001$.

[8] R. Goldstein, Z.A. Luthey-Schulten, and P.G. Wolynes. Protein tertiary structure recognition using optimized hamiltonians with local interactions. Proc. Natl. Acad. Sci. USA, 89:9029-9033, 1992.

[9] P.D. Thomas and K.A. Dill. An iterative method for extracting energy-like quantities from protein 
structures. Proc Natl Acad Sci USA, 93:1162811633, 1996.

[10] U. Bastolla, J. Farwer, E.W. Knapp, and M. Vendruscolo. How to guarantee optimal stability for most representative structurs in the protein data bank. Proteins, 44:79-96, 2001.

[11] Banavar J.R. Dima R.I. and Maritan A. Scoring functions in protein folding and design. Protein Sci., 9:812-819, 2000.

[12] C. Micheletti, F. Seno, J.R. Banavar, and A. Maritan. Learning effective amino acid interactions through iterative stochastic techniques. Proteins, 42(3):422-431, 20001.

[13] H. Edelsbrunner and E.P. Mücke. Threedimensional alpha shapes. ACM Trans Graphics, 13:43-72, 1994 .

[14] J. Liang, H. Edelsbrunner, P. Fu, P.V. Sudhakar, and S. Subramaniam. Analytical shape computing of macromolecules I: Molecular area and volume through alpha-shape. Proteins, 33:1-17, 1998.

[15] Wernisch L., Hunting M., and Wodak S.J. Identification of structural domains in proteins by a graph heuristic. Proteins., 35(3):338-52, 1999.

[16] D. Riddle, J. Santiago, V. Grantcharova, and D. Baker. Functional rapidly folding proteins from simplified amino acid sequences. Nature Structural Biology, 4:805-809, 1997.

[17] T.M.A. Fink and R.C. Ball. How many conformations can a protein remember? Phys. Rev. Lett., 87:198103-1-198103-4, 2001.

[18] J. Liang, H. Edelsbrunner, P. Fu, P.V. Sudhakar, and S. Subramaniam. Analytical shape computing of macromolecules II: Identification and computation of inaccessible cavities inside proteins. Proteins, 33:18-29, 1998.

[19] H. Edeslbrunner, M. Facello, and J. Liang. On the definition and the construction of pockets in macromolecules. Disc. Appl. Math., 88:18-29, 1998.

[20] J. Liang, H. Edelsbrunner, and C. Woodward. Anatomy of protein pockets and cavities: Measurement of binding site geometry and implications for ligand design. Protein Sci, 7:1884-1897, 1998.
[21] H. Edelsbrunner, D. Letscher, and A. Zomorodian. Topological persistence and simplification. In Proc. 41st Ann. IEEE Sympos. Found Comput. Sci., pages 454-463, 2000.

[22] H. Edelsbrunner and A. Zomorodian. Computing linking numbers in a filtration. In Algorithms in Bioinformatics (LNCS 2149), pages 112-127. Springer, Berlin, 2001.

[23] H. Edelsbrunner, J. Harer, and A. Zomorodian. Hierarchical Morse complexes for piecewise linear 2-manifolds. In Proc. 17th Ann. ACM Sympos. Comput. Geom., pages 70-79, 2001.

[24] J.R. Bienkowska, R.G. Rogers, and T.F. Smith. Filtered neighbors threading. Proteins, 37:346359, 1999.

[25] W.R. Taylor. Multiple sequence threading: an analysis of alignment quality and stability. $J$. Mol. Biol., 269:902-943, 1997.

[26] H. Edelsbrunner, M. Facello, P. Fu, and J. Liang. Measuring proteins and voids in proteins. In Proc. 28th Ann. Hawaii Int'l Conf. System Sciences, volume 5, pages 256-264, Los Alamitos, California, 1995. IEEE Computer Scociety Press.

[27] K.P. Peters, J. Fauck, and C. Frömmel. The automatic search for ligand binding sites in proteins of known three-dimensional structure using only geometric criteria. J. Mol. Biol., 256:201$213,1996$.

[28] J. Liang and K.A. Dill. Are proteins wellpacked? Biophys. J., 81(2):751-766, 2001.

[29] J. Liang and S. Subranmaniam. Computation of molecular electrostatics with boundary element methods. Biophys. J., 73:1830-1841, 1997.

[30] J. Liang and M.P. McGee. Mechanisms of coagulation factor Xa inhibition by antithrombin. Correlation between hydration structure and water transfer during reactive loop insertion. Biopys. J., 75:573-582, 1998.

[31] J. Tsai, R. Taylor, C. Chothia, and M. Gerstein. The packing density in proteins: Standard radii and volumes. J. Mol. Biol., 290:253-266, 1999.

[32] J. Singh and J.M. Thornton. Atlas of protein side-chain interactions, volume I, II. IRL Press, Oxford, 1992. 
[33] J. Skolnick, A. Kolinski, and A. Ortiz. Derivation of protein-specific pair potentials based on weak sequence fragment similarity. Proteins, $38: 3-16,2000$.

[34] L. Adamian and J. Liang. Helix-helix packing and interfacial pairwise interactions of residues in membrane proteins. J. Mol. Biol., 311:891907, 2001.

[35] B. Efron and R.J. Tibshirani. An introduction to the bootstrap. Chapman \& Hall, New York, 1993.

[36] A.C. Davison and D.V. Hinkley. Bootstrap methods and their applications. Cambridge University Press, Cambridge, U.K., 1997.

[37] Hobohm U., Scharf M., Schneider R., and Sander C. Selection of a representative set of structures from the brookhaven protein data bank. Protein Science, 1:409-417, 1992.

[38] Hobohm U. and Sander C. Enlarged representative set of protein structures. Protein Science, 3:522-524, 1994.

[39] L. Kaufman and P.J. Rousseeuw. Finding groups in data. John Wiley \& Sons, 1990.

[40] Henikoff S. and Henikoff J.G. Amino acid substitution matrices from protein blocks. Proc. Natl. Acad. Sci., 89:10915-10919, 1992.

[41] Murphy L.R., Wallqvist A., and Levy R.M. Simplified amino acid alphabets for protein fold recognition and implications for folding. Protein Eng., 13(3):149-152, 2000.

[42] L. Holm and C. Sander. Evaluation of protein models by atomic solvation preference. J. Mol. Biol., 225:93-105, 1992.

[43] B. Park and M. Levitt. Energy functions that discriminate $\mathrm{x}$-ray and near-native folds from well-constructed decoys. J. Mol. Biol., 258:367392, 1996.

[44] R. Samudrala, Y. Xia, M. Levitt, and E.S. Huang. A combined approach for ab initio construction of low resolution protein tertiary structures from sequence. Pac. Symp. Biocomput., 1999.

[45] Y. Xia and M. Levitt. Extracting knowledgebased energy functions from protein structures by error rate minimization: Comparison of methods using lattice model. J Chem. Phys., 113:9318-9330, 2000.

[46] B. Park, E.S. Huang, and M. Levitt. Factors affecting the ability of energy functions to discriminate correct from incorrect folds. J. Mol. Biol., 266:831-846, 1997.

[47] R. Samudrala and M. Levitt. Decoys ' $R$ ' us: a database of incorrect conformations to improved protein structure prediction. Protein Sci., 9:1399-1401, 2000.

[48] Levitt M. and Lifson S. Rifinement of protein conformations using a macromolecular energy minimization procedure. J. Mol. Biol., 46:269279, 1969.

[49] M. Levitt. Energy refinement of hen egg-white lysozyme. J. Mol. Biol., 82:392-420, 1974.

[50] M. Levitt. Molecular dynamics of native protein: i. computer simulation of trajectories. J. Mol. Biol., 168:595-620, 1983.

[51] Levitt M., Hirshberg M., Sharon R., and Daggett V. Potential energy function and parameters for simulations of the molecular dynamics of proteins and nucleic acids in solution. Comp. Phys. Comm., 91:215-231, 1995.

[52] Fletcher R. A new approach to variable metric algorithms. Comput. J., 13:317-322, 1970.

[53] D. Tobi and R. Elber. Distance-dependent, pair potential for protein folding: Results from linear optimization. Proteins, 41:40-46, 2000.

[54] S. Miyazawa and R. Jernigan. Residue-residue potentials with a favorable contact pair term and an unfavorable high packing density term. $J$. Mol. Biol., 256:623-644, 1996.

[55] M.R. Betancourt and D. Thirumalai. Pair potentials for protein folding: Choice of reference states and sensitivity of predicted native states to variations in the interaction schemes. Protein Sci., 8:361-369, 1999.

[56] K. W. Plaxco, K. T. Simons, and D. Baker. Contact order, transition state placement, and the refolding rates of single domain proteins. J. Mol. Biol., 277:985-994, 1998. 
[57] K. Yue and K.A. Dill. Inverse protein folding problem: Designing polymer sequences. Proc. Natl. Acad. Sci. USA., 89:4163-4167, 1992.

[58] E.I. Shakhnovich and A.M. Gutin. Engineering of stable and fast-folding sequences of model proteins. Proc. Natl. Acad. Sci. USA., 90:71957199, 1993.

[59] J.M. Deutsch and T. Kurosky. New algorithm for protein design. Phys. Rev. Lett., 76(2):323$326,1996$.

[60] H. Li, R. Helling, C. Tang, and N. Wingreen. Emergence of preferred structures in a simple model of protein folding. Science, 273:666-669, 1996.

[61] R. Mélin, H. Li, N. Wingreen, and C. Tang. Designability, thermodynamic stability, and dynamics in protein folding: a lattice model study. $J$. Chem. Phys., 110:1252-1262, 1999.

[62] Sander C. and Schulz G.E. Degeneracy of the information contained in amino acid sequences: evidence from overlaid genes. J. Mol. Evol., 13(3):245-52, 1979.

[63] Heinz D.W., Baase W.A., and Matthews B.W. Folding and function of a t4 lysozyme containing 10 consecutive alanines illustrate the redundancy of information in an amino acid sequence. Proc. Natl. Acad. Sci., 89:3751-3755, 1992.

[64] Riddle D.S., Santiago J.V., Bray-Hall S.T., Doshi N., Grantcharova V.P., Yi Q., and Baker D. Functional rapidly folding proteins from simplified amino acid sequences. Nat. Struct. Biol., 4(10):805-9, 1997.

[65] Wang J. and Wang W. A computational approach to simplifying the protein folding alphabet. Nat. Struct. Biol., 6(11):1033-8, 1999.

[66] M.S. Cline, K. Karplus, R.H. Lathrop, T.F. Smith, and R.G. Rogers Jr. Informationtheoretical dissection of pairwise contact potentials. Proteins, 49:7-14, 2002.

[67] R.K. Singh, A. Tropsha, and I.I. Vaisman. Delaunay tessellation of proteins: four body nearest-neighbor propensities of amino-acid residues. J. Comp. Bio., 3:213-221, 1996.
[68] W. Zheng, S.J. Cho, I.I. Vaisman, and A. Tropsha. A new approach to protein fold recognition based on Delaunay tessellation of protein structure. In R.B. Altman, A.K. Dunker, L. Hunter, and T.E. Klein, editors, Pacific Symposium on Biocomputing'97, pages 486-497, Singapore, 1997. World Scientific.

[69] C.W. Carter Jr, B.C. LeFebvre, S.A. Cammer, A. Tropsha, and M.H. Edgell. Four-body potentials reveal protein-specific correlations to stability changes caused by hydrophobic core mutations. J. Mol. Biol., 311:625-638, 2001. 